\title{
Clinical features and genotype-phenotype correlation analysis in patients with ATL 1 mutations: A literature reanalysis
}

\author{
Guo-hua Zhao ${ }^{1,2}$ and Xiao-min Liu ${ }^{3^{*}}$
}

\begin{abstract}
Background: The hereditary spastic paraplegias (HSPS) are a group of clinically and genetically heterogeneous disorders. Approximately 10\% of the autosomal dominant (AD) HSPs (ADHSPs) have the spastic paraplegia 3A (SPG3A) genotype which is caused by ATL1 gene mutations. Currently there are more than 60 reported ATL1 gene mutations and the genotype-phenotype correlation remains unclear. The study aims to investigate the genotypephenotype correlation in SPG3A patients.

Methods: We performed a reanalysis of the clinical features and genotype-phenotype correlations in 51 reported studies exhibiting an ATL1 gene mutation.

Results: Most HSPs-SPG3A patients exhibited an early age at onset (AAO) of $<10$ years old, and showed an autosomal dominant pure spastic paraplegia. We found that 14\% of the HSPs-SPG3A patients presented complicated phenotypes, with distal atrophy being the most common complicated symptom. The AAO of each mutation group was not statistically significant $(P>0.05)$. The mutational spectrum associated with ATL1 gene mutation is wide, and most mutations are missense mutations, but do not involve the functional motif of ATL1 gene encoded atlastin-1 protein.
\end{abstract}

Conclusions: Our findings indicate that there is no clear genotype-phenotype correlation in HSPs-SPG3A patients. We also find that exons 4, 7, 8 and 12 are mutation hotspots in ATL1 gene.

Keywords: Hereditary spastic paraplegia, SPG3A, Age at onset, ATL1, Mutation, Genotype-phenotype correlation

\section{Background}

The hereditary spastic paraplegias (HSPs) are a group of clinically heterogeneous neurological disorders, which are classified into "pure" or "complicated" HSP according to the clinical features. The pure HSP is defined by progressive spasticity and weakness limited to the lower limbs, while the complicated HSP may include other neurological manifestations such as optic atrophy, retinal pigmentation, seizures, deafness, neuropathy and mental retardation. In the clinic, HSPs can also be classified into early onset (mainly $1^{\text {st }}$ decade of life) and late onset (between the $2^{\text {nd }}$ and $4^{\text {th }}$ decade) type. The main

\footnotetext{
* Correspondence: greenfield008@sina.com

${ }^{3}$ Department of Neurology, Qianfoshan Hospital, Shandong University, Jinan 16766, China

Full list of author information is available at the end of the article
}

pathological changes of HSP include the axonal degeneration of the corticospinal tracts and back column $[1,2]$.

Genetic mutations are the main cause of HSPs and there are currently over 72 spastic paraplegia genes or genetic loci (designated SPG1-SPG72 genetic type in order of their discovery) in which mutations can occur [3]. HSPs can be inherited as autosomal dominant (AD), autosomal recessive (AR) or X-linked trait or a spastic paraplegia syndrome. Among identified mutations, approximately $40 \%$ of definite autosomal dominant pure HSP mutations are in the spastic paraplegia 4 (SPG4/ $S P A S T)$ gene which encodes the spastin protein $[4,5]$.

SPG3A is the second most common type of HSP which accounts for approximately $10 \%$ of autosomal dominant HSP [6] and is caused by mutations in the atlastin-1 (ATL1) gene. The atlastin-1 protein is a member of the dynamin family of large guanosine 
triphosphatases (GTPases) which contains three conserved motif-P loops (74GAFRKGKS81), RD (217RD) and DxxG (146DTQG) which are characteristic regions for guanylate binding/GTPase active sites [6]. HSPsSPG3A phenotype (HSPs with ATL1 gene mutations) was generally a pure HSP with age at onset (AAO) less than 10 years old [6]. Patients characterize progressive bilateral and mostly symmetric lower extremity weakness and spasticity.

Currently there are more than 60 different ATL1 gene mutations described, including numerous missense, small deletion, small insertion and splice site mutations, as well as whole exon deletions [6-56]. However, the genotype-phenotype correlation remains unclear [13]. In this study, we perform a reanalysis of all published studies $(n=51)$ to identify the clinical features and then genotype-phenotype correlations in HSPs caused by ATL1 gene mutations.

\section{Methods}

We conducted a literature search using databases from PubMed (http://ncbi.nlm.nih.gov/pubmed) and the China National Knowledge Infrastructure (CNKI) (http://cnki.net) with the keyword "SPG3A" or "ATL1", which resulted in 51 articles describing ATL1 gene mutations [6-56]. We collected information related to the age at onset (AAO), age at examination, pure or complicated form, involvement of upper and lower limbs, Babinski signs, urinary urgency and other symptoms or signs for individually affected patients directly from relevant papers. Asymptomatic individuals were also included, but excluded from the analysis of AAO and pure or complicated form. Patients with elderly sensory neuropathy caused by ATL1 gene mutations were excluded in this study $[57,58]$. We reanalysed the clinical and genetic data in ATL1 gene mutant patients and performed a correlation analysis of AAO with mutational class in ATL1 gene. Comparisons of data were performed using two-way ANOVA. Tests were considered statistically significant for $P<0.05$.

\section{Results}

The patients' clinical information and the ATL1 gene mutation of 51 reports are summarized in Additional file 1: Table S1. The published studies contain data for 142 families with known ATL1 gene mutations. These 142 families included 130 (91.54\%) autosomal dominant HSP (ADHSP) families, 10 (7.04\%) sporadic families, one $(0.70 \%)$ ARHSP family, and one $(0.70 \%)$ family with unknown inheritance mode. Gender information was available in 151 patients, including 88 male patients and 63 female patients (ratio is 1.40:1). The main clinical features included lower spasticity $(99.68 \%, 313 / 314)$, upper spasticity $(10.03 \%, 30 / 299)$, Babinski sign $(87.83 \%, 231 /$
$263)$ and urinary urgency $(16.37 \%, 38 / 232)$. AAO data were available in 355 subjects from infancy to the seventh decade, in which 301 (84.79\%) patients had AAO < 10 years old, whereas 54 (15.21\%) patients had AAO $>10$ years old. Patient information for pure or complicated type was available in 440 patients, including 378 (85.90\%) pure HSP and $62(14.10 \%)$ complicated HSP patients. Distal atrophy or neuropathy is the most common symptom in patients with complicated SPG3A gene mutations $(69.35 \%, 43 / 62)$. In addition, $15 / 142$ families (10.56\%) showed incomplete penetrance.

In total, there were 61 different types of mutations reported, which were divided into five broad groups: 130 (91.54\%) families had missense mutations (54 types), six (4.23\%) had small insertions (4 types), four had (2.82\%) small deletions (2 types), one $(0.70 \%)$ had presumed splice site mutation (1 type), and one $(0.70 \%)$ had whole exon deletion ( 1 type). The mutations were located in exon $3(0.70 \%, n=1)$, exon $4(11.26 \%, n=16)$, exon 5 $(1.41 \%, n=2)$, exon $6(0.70 \%, n=1)$, exon $7(24.65 \%, n=$ $35)$, exon $8(12.68 \%, n=18)$, exon $9(1.41 \%, n=2)$, exon $10(5.63 \%, n=8)$, exon $11(1.41 \%, n=2)$, exon 12 $(38.73 \%, n=55)$, exon $13(0.70 \%, n=1)$, and intron 1 $(0.70 \%, n=1)$. A total of $124(87.32 \%)$ mutations were found in exons $4,7,8$ and 12 . No mutations were detected in exons 1, 2 and 14. Figure 1 shows the locations of ATL1 gene mutations and the number of mutations found in the families. Most mutations did not involve in the functional motif of atlastin-1, except R217Q, c.35$3 \mathrm{C}>\mathrm{T}$ and exon 4 deletion $[7,13,40]$. The most commonly reported mutations were $\mathrm{R} 239 \mathrm{C}(n=31)$ and R495W ( $n=14)$. All the published mutations are listed in Ensemble database (ensemble.org).

The AAOs of each mutation group are summarized in Table 1. The patients with missense mutations had a slightly lower AAO, however this difference is not statistically significant $(F=1.273, P=0.282)$. The patients with splice site mutation and exon deletion were not included in the statistics because there was only one patient in each group.

\section{Discussion}

HSPs-SPG3A patients account for approximately $10 \%$ of ADHSP. Although several large cohorts of patients with mutant ATL1 gene were reported [17, 21, 27, 29, 32, 36, $45,50,54,56]$, a genotype-phenotype correlation still remains unclear. Here, we reanalysed the observations on 142 families and confirmed three previously reported observations. First, we find that most HSPs-SPG3A patients exhibiting early AAO and autosomal dominant pure spastic paraplegia there have a wide mutational spectrum associated with ATL1 gene mutations. Second, we find that most mutations are missense but do not involve the functional motifs of atlastin-1. Third, we note 


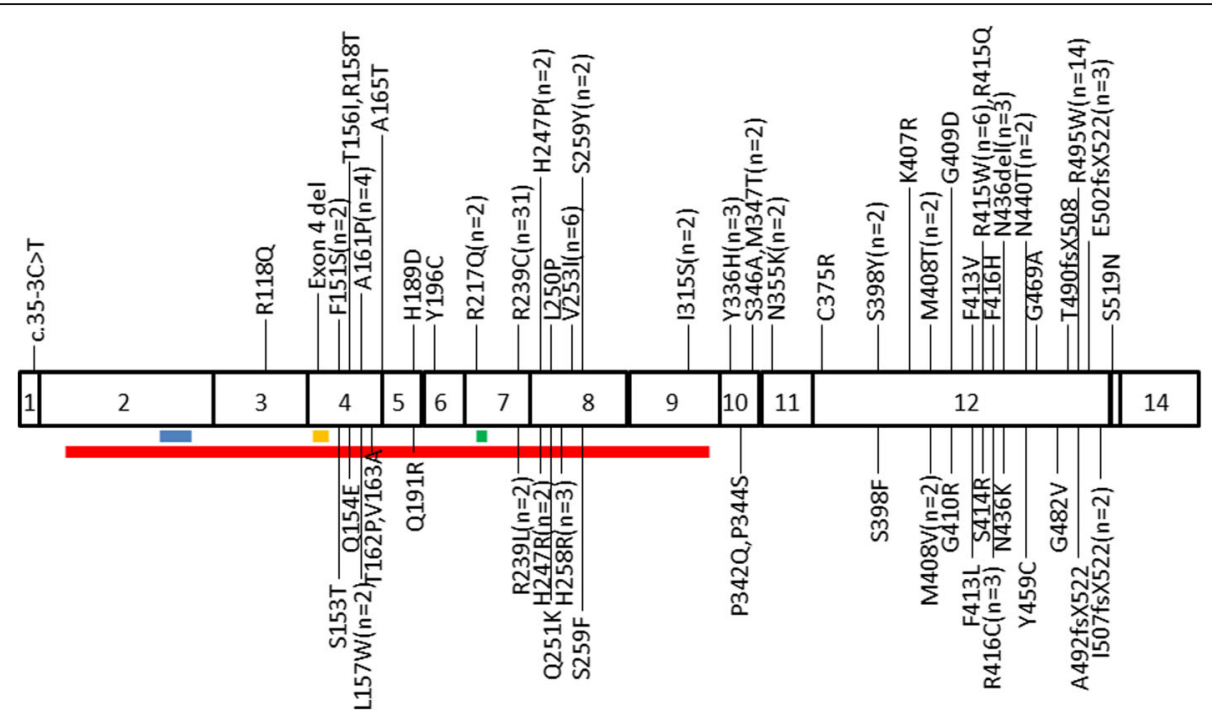

Fig. 1 The ATL1 mutation spectrum in CDS. Red line indicates the GBp/Ras-like GTPase domain; blue line shows the P-loop domain; orange line displays the DxxG domain; green line indicates the RD domain. $n$ correlates with the number (if $\geq 2$ ) of families containing ATL1 mutations

that exon 4, 7, 8 and 12 might be mutation hotspots. Additionally, we found that the complicated type was prevalent in HSPs-SPG3A patients, and that distal atrophy or neuropathy is the most common complicated symptoms.

ATL1 gene mutations are thought to be the most common cause of hereditary spastic paraplegia with an AAO $<10$ years [17]. Our re-analysis of the 51 reported studies showed that $84.79 \%$ patients exhibited early AAO ( $<10$ years), but we found that $15.21 \%$ patients had a later AAO ( $>10$ years). Therefore, ATL1 gene mutation analysis should not be limited to early onset HSP [11, 34].

Our re-analysis also showed no correlation between $\mathrm{AAO}$ and mutational classes. More studies with larger sample sizes may be required to resolve this issue because of the limited number of small insertion, small deletion, splice site mutation and exon deletion. In addition, there was variability of AAOs between families with the same mutation, even within the same family. Some members in different families exhibiting the same

Table 1 The comparison of AAOs between different kinds of mutations

\begin{tabular}{llll}
\hline Mutation type & No. of families & No. of subjects & $\begin{array}{l}\text { Mean age at } \\
\text { onset (years) }\end{array}$ \\
\hline Missense & 109 & 271 & $7.47 \pm 11.97$ \\
Small insertion & 6 & 10 & $13.60 \pm 12.39$ \\
Small deletion & 4 & 12 & $8.25 \pm 11.87$ \\
Splice site & 1 & 1 & 44 \\
Exon deletion & 1 & 1 & Not available \\
\hline
\end{tabular}

$F=1.273, P=0.282$. No.: number mutation had different AAOs. For example, the AAOs for the A161P ATL1 gene mutation could be childhood or age 45-55 in different families [11]. We found that both childhood-onset HSP and late onset HSP (after age 40 years) occurred in the same family with A161P mutation [21]. Furthermore, intrafamilial variability in AAO varied from eight to 28 years in a family with R495W mutation [21]. One family member with complicated HSP showed AAOs $>30$ years, whereas another family member with pure HSP presented AAO at puberty, but both members had a R416C mutation in the ATL1 gene, suggesting a clear intrafamilial variability. Hedera et al. reported a family in which patients had a variable AAO from five to 39 years, and two subjects were functionally asymptomatic despite abnormalities in neurological examinations [15]. Patients in an ADHSP family carrying the ATL1 R416C mutation were found to have variable clinical characteristics, both the pure phenotype with early onset and the complicated phenotype with later onset [41]. Differences of AAO and clinical features between families with the same mutation or in the same family might be due to variability in expression of this mutation or maybe to related to other genetic or epigenetic factors [11, 18, 34]. Overall, the comparison of the clinical data for all ATL1 gene mutation families failed to reveal any genotype-phenotype correlation as demonstrated in other types of ADHSP [13].

ATL1 gene was commonly thought to be associated with pure spastic paraplegia manifesting as lower limb spasticity, decreased vibration sense in the lower limbs, and sphincter disturbances. Information for pure or complicated HSPs were available in 440 patients, including 378 (85.90\%) pure patients and 62 (14.10\%) 
complicated patients. Our re-analysis suggests that most ATL1 gene mutations usually display a pure phenotype, but ATL1 gene mutation can also been found in patients with complicated phenotype of HSPs. The complicated symptoms of HSPs-SPG3A patients included seizure, optic atrophy, sensory impairment, mental retardation, ataxia, distal atrophy and peripheral axonal neuropathy (Additional file 1: Table S1). Additionally, we found that distal atrophy is the most common symptom in complicated HSPs-SPG3A patients (69.35\%, 43/62).

The early-onset and relatively non-progressive nature of lower extremity spasticity in HSPs-SPG3A patients closely resembles symptoms of patients with spastic diplegic cerebral palsy. Because of this, many HSPsSPG3A cases have been misdiagnosed as cerebral palsy even when there is no antecedent of a perinatal sentinel event and no lesions detected on brain imaging [10, 28, 31, 47]. However, reaching a HSP diagnosis in paediatric cases is challenging, especially in the absence of a positive family history. However, the occurrence of a de novo ALT1 gene mutation must be considered in patients with spastic diplegic cerebral palsy, when other causes can not be identified.

Disease severity in HSPs-SPG3A patients is most commonly mild, although the severity of spasticity increases with disease duration. In general, the onset of disease symptoms in children has a long phase of relatively slow progression. In many cases, symptoms remain unchanged up to old age. Additionally, there are some asymptomic cases that contain the ATL1 gene mutations. Incomplete penetrance has been previously reported in 10.56\% HSPs families [8, 12, 15, 24, 27, 45]. The scarce penetrance of the mutations favours a modulator gene or strong epigenetic factor hypothesis, which may influence the phenotype. However, previous reports have shown the existence of some severe symptoms in HSPs-SPG3A patient. For example, Haberlova et al. reported a HSPs-SPG3A patient with a severe and early complicated phenotype, which was caused by the M408T mutation in ATL1 gene [25]. Furthermore, a de novo G409D mutation in the ATL1 gene exhibited an extremely severe spastic paraplegia combined with general hypertonia and hypokinesia since the neonatal period in one patient [47].

Linkage analysis suggests that mutations in the ATL1 gene account for approximately $10 \%$ of ADHSP. The reported frequency of ATL1 gene mutations varied from 2.9 to $38.7 \%$ though most studies reported a frequency of less than $15 \%$. For example, we found that there were eight studies which reported a frequency of ATL1 gene mutations less than 15\%: 2.9\% [27] in ADHSP families, $3.7 \%$ in ADSHP probands [45], 4.2\% in HSP families [36], 8.3\% in unrelated early onset pure ADHSP families [9], 6.6\% in a heterogeneous population including both pure and complicated HSP phenotypes [21], 8.6\% in ADHSP families [29], 11.3\% in the ADHSP families [32], and $11.7 \%$ in ADHSP probands [44]. We also found four studies which reported a higher frequency of ATL1 gene mutations: $20.0 \%$ in pure HSP [55], 20.0\% (3/15) in early onset autosomal dominant HSP [13], 38.5\% in SPG4negative pure ADHSP families [11], and $38.7 \%$ in pure ADHSP families [15]. We also found that there was a difference in the frequency of ATL1 gene mutations reported with different AAOs. For example, Namekawa et al. reported that the frequency of ATL1 gene mutation in ADHSP was $6.6 \%$, whereas the frequency was $13.5 \%$ in ADHSP families with onset before age 20, and it increased to $31.8 \%$ in ADHSP families with onset before age 10 [17]. The frequency variation may be caused by the differences in ethical criteria, number of patients and inclusion criteria, such as pure and complicated phenotypes of the patients, AAOs and SPAST mutations.

This study found that most ATL1 gene mutations were located at exons 4, 7, 8 and 12, which is consistent with a previous study [29], which suggests that these exons should be given priority when performing molecular diagnosis. In addition, $\mathrm{R} 239 \mathrm{C}(n=31)[6,9,11,13,15$, $17,21,22,27-29,31,32,36,39,45,51,53,54,56]$ and R495W ( $n=14)$ [15-17, 21, 32, 36, 43, 50, 52, 54] mutations were the most commonly reported mutations in all studied families. Zhao et al. reported that the three families with R239C mutations were not apparently related and haplotype analysis did not exclude a distant founder effect [6]. Namekawa et al. reported that the R495W mutation could occur by independent mutational events [59]. Genetic testing should be performed in HSPs patients with very early-onset pure spastic paraplegia.

It is still not understood how the atlastin-1 protein functions and it is also unclear how autosomal dominant mutations in ATL1 gene lead to the degeneration of upper motor neurons. In our analysis of the literature we found that all ATL1 gene mutations except 3 (R217Q, c.35-3C < $\mathrm{T}$, and exon 4 deletion) fell outside the GTPase-related motifs or the conserved motifs identified in the ATL1 gene sequence which are thought to alter the structure of atlastin1 and its interaction with other proteins $[6,11,13,16]$. We find that most mutations are missense which suggests a gain-of-function pathogenic mechanism that is dependent on the position of the mutation, gene modifier and environmental factors [32]. This is supported by studies using yeast two-hybrid assay and co-immunoprecipitation of wild-type and p.del436N atlastin proteins which show that the p.del $436 \mathrm{~N}$ mutant protein can still oligomerize with wild-type atlastin, supporting a loss-of-function disease mechanism [23]. Atlastin-1 interacts with spastin (SPG4), suggesting that they may be a part of a common biological cascade whose disruption can result in motor neuron death [60]. 


\section{Conclusions}

Our reanalysis demonstrates that most HSPs-SPG3A patients exhibited a pure autosomal dominant HSP with early AAO. The causal ATL1 gene mutations are missense mutations and exons 4, 7, 8 and 12 should be prioritized for genetic testing. We find that there is no clear genotype-phenotype correlation.

\section{Additional file}

Additional file 1: Table S1. Correlation between clinical and genetic

characteristics in patients with mutant ATL1. (DOC 562 kb)

\section{Abbreviations}

AAO: Age at onset; AD: Autosomal dominant; ADHSP: Autosomal dominant HSP; AR: Autosomal recessive; GTPases: Guanosine triphosphatases; HSP: Hereditary spastic paraplegias; SPG3A: Spastic paraplegia 3A

\section{Acknowledgements}

Not applicable.

\section{Funding}

This study was supported by the National Scientific Foundations of China (81000484), Natural Scientific Fundation of Zhejiang Province (LY17H090002), the Research Fundation of Zhejiang Health (2008QN017, 2016144072), the Natural Science Foundation of Shandong Province (ZR2013HQ016), and the Key Research and Development Project of Shandong Province (2015GGH318011)

\section{Availability of data and materials}

Data sharing not applicable to this article as no datasets were generated or analyzed during the current study.

\section{Authors' contributions}

All authors read and approved the final manuscript. GZ and XL conceived, designed and performed the paper. GZ revised the paper.

\section{Competing interests}

The authors declare that they have no competing interest.

\section{Consent for publication}

Not relevant.

\section{Ethics approval and consent to participate} Not relevant.

\section{Publisher's Note}

Springer Nature remains neutral with regard to jurisdictional claims in published maps and institutional affiliations.

\section{Author details}

'Department of Neurology, Second Affiliated Hospital, School of Medicine, Zhejiang University, Hangzhou 310009, China. ${ }^{2}$ Department of Neurology, Fourth Affiliated Hospital, School of Medicine, Zhejiang University, Yiwu 322000, China. ${ }^{3}$ Department of Neurology, Qianfoshan Hospital, Shandong University, Jinan 16766, China.

Received: 20 February 2017 Accepted: 28 March 2017

Published online: 04 April 2017

\section{References}

1. Harding AE. Classification of the hereditary ataxia and paraplegias. Lancet. 1983;1(8334):1151-5.

2. Mc Dermott CJ, et al. Hereditary spastic paraparesis: a review of new developments. J Neurol Neurosurg Psychiatry. 2000;69(2):150-60.

3. Gan-Or Z, et al. Mutations in CAPN1 cause autosomal- recessive hereditary spastic paraplegia. Am J Hum Genet. 2016;98(5):1038-46.
4. Hazan J, et al. Spastin, a new AAA protein, is altered in the most frequent form of autosomal dominant spastic paraplegia. Nat Genet. 1999;23(3): 296-303.

5. Tallaksen CM, Durr A, Brice A. Recent advances in hereditary spastic paraplegia. Current Opinion Neurol. 2001;14:457-63.

6. Zhao $X$, et al. Mutations in a newly identified GTPase gene cause autosomal dominant hereditary spastic paraplegia. Nat Genet. 2001;29(3):326-31.

7. Muglia M, Magariello A, Nicoletti G. Further evidence that SPG3A gene mutations cause autosomal dominant hereditary spastic paraplegia. Ann Neurol. 2002;51(6):794-5

8. Tessa A, Casali C, Damiano M. SPG3A: An additional family carrying a new atlastin mutation. Neurology. 2002;59(12):2002-5.

9. Wilkinson PA, et al. SPG3A mutation screening in English families with early onset autosomal dominant hereditary spastic paraplegia. J Neurol Sci. 2003; 216(1):43-5.

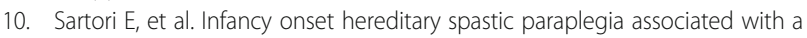
novel atlastin mutation. Neurology. 2003;61(4):580-1.

11. Sauter SM, et al. Novel mutations in the Atlastin gene (SPG3A) in families with autosomal dominant hereditary spastic paraplegia and evidence for late onset forms of HSP linked to the SPG3A locus. Hum Mutat. 2004; 23(1):98.

12. D'Amico A, et al. Incomplete penetrance in an SPG3A-linked family with a new mutation in the atlastin gene. Neurology. 2004;62(11):2138-9.

13. Abel A, et al. Early onset autosomal dominant spastic paraplegia caused by novel mutations in SPG3A. Neurogenetics. 2004;5(4):239-43.

14. Hedera $P$, et al. Novel mutation in the SPG3A gene in an African American family with an early onset of hereditary spastic paraplegia. Arch Neurol. 2004:61(10):1600-3.

15. Dürr A, et al. Atlastin1 mutations are frequent in young-onset autosomal dominant spastic paraplegia. Arch Neurol. 2004;61(12):1867-72.

16. Scarano V, et al. The R495W mutation in SPG3A causes spastic paraplegia associated with axonal neuropathy. J Neurol. 2005;252(8):901-3.

17. Namekawa M, et al. SPG3A is the most frequent cause of hereditary spastic paraplegia with onset before age 10 years. Neurology. 2006;66(1):112-4.

18. Rainier $\mathrm{S}$, et al. De novo occurrence of novel SPG3A/atlastin mutation presenting as cerebral palsy. Arch Neurol. 2005:63(3):445-7.

19. Chen $\mathrm{SQ}$, et al. Severe hereditary spastic paraplegia caused by a de novo SPG3A mutation. Sci China. 2005:51(15):1854-6. In Chinese.

20. Matsui $M$, et al. A novel mutation in the SPG3A gene (atlastin) in hereditary spastic paraplegia. J Neurol. 2007;54(7):972-4.

21. Ivanova $\mathrm{N}$, et al. Hereditary spastic paraplegia $3 \mathrm{~A}$ associated with axonal neuropathy. Arch Neurol. 2007;64(5):706-13.

22. $\mathrm{Li} \mathrm{XH}$, et al. A SPG3A mutation with a novel foot phenotype of hereditary spastic paraplegia in a Chinese Han family. Chin Med J (Engl). 2007;120(9): 834-7.

23. Meijer IA, et al. Characterization of a novel SPG3A deletion in a FrenchCanadian family. Ann Neurol. 2007;61(6):599-603.

24. Ming L. SPG3A-hereditary spastin paraplegia with genetic anticipation and incomplete penetrance. Zhonghua Yi Xue Yi Chuan Xue Za Zhi. 2007;24(1): 15-8. In Chinese.

25. Haberlová J, et al. Extending the clinical spectrum of SPG3A mutations to a very severe and very early complicated phenotype. J Neurol. 2008;255(6): 927-8.

26. Loureiro $\mathrm{L}$, et al. Novel SPG3A and SPG4 mutations in dominant spastic paraplegia families. Acta Neurol Scand. 2009:119(2):113-8.

27. Svenstrup $K$, et al. Sequence variants in SPAST, SPG3A and HSPD1 in hereditary spastic paraplegia. J Neurol Sci. 2009;284(1-2):90-5.

28. Chan KY, et al. Hereditary spastic paraplegia: identification of an SPG3A gene mutation in a Chinese family. Hong Kong Med J. 2009;15(4):304-7.

29. Smith BN, et al. Four novel SPG3A/atlastin mutations identified in autosomal dominant hereditary spastic paraplegia kindreds with intra-familial variability in age of onset and complex phenotype. Clin Genet. 2009;75(5):485-9.

30. Fusco C, et al. Hereditary spastic paraplegia and axonal motor neuropathy caused by a novel SPG3A de novo mutation. Brain Dev. 2010;32(7):592-4.

31. Kwon MJ, et al. Clinical and genetic analysis of a Korean family with hereditary spastic paraplegia type 3. Ann Clin Lab Sci. 2010;40(4):375-9.

32. Alvarez $\mathrm{V}$, et al. Mutational spectrum of the SPG4 (SPAST) and SPG3A (ATL1) genes in Spanish patients with hereditary spastic paraplegia. BMC Neurol. 2010;10:89.

33. de Leva MF, et al. Complex phenotype in an Italian family with a novel mutation in SPG3A. J Neurol. 2010;257(3):328-31. 
34. Orlacchio A, et al. Late-onset hereditary spastic paraplegia with thin corpus callosum caused by a new SPG3A mutation. J Neurol. 2011;258(7): 1361-3.

35. Al-Maawali A, et al. Hereditary spastic paraplegia associated with axonal neuropathy: a novel mutation of SPG3A in a large family. J Clin Neuromuscul Dis. 2011;12(3):143-6.

36. McCorquodale III DS, et al. Mutation screening of spastin, atlastin, and REEP1 in hereditary spastic paraplegia. Clin Genet. 2011;79(6):523-30.

37. Magariello A, et al. The p.Arg416Cys mutation in SPG3a gene associated with a pure form of spastic paraplegia. Muscle Nerve. 2012;45(6):919-20.

38. Fusco C, et al. Very early onset and severe complicated phenotype caused by a new spastic paraplegia 3A gene mutation. J Child Neurol. 2012;27(10): 1348-50.

39. Sulek A, et al. Screening for the hereditary spastic paraplaegias SPG4 and SPG3A with the multiplex ligation-dependent probe amplification technique in a large population of affected individuals. Neurol Sci. 2012; 34(2):239-42.

40. Terada T, et al. SPG3A-linked hereditary spastic paraplegia associated with cerebral glucose hypometabolism. Ann Nucl Med. 2013;27(3):303-8.

41. de Bot ST, et al. ATL1 and REEP1 mutations in hereditary and sporadic upper motor neuron syndromes. J Neurol. 2013:260(3):869-75.

42. Varga RE, et al. Do not trust the pedigree: reduced and sex-dependent penetrance at a novel mutation hotspot in ATL1 blurs autosomal dominant inheritance of spastic paraplegia. Hum Mutat. 2013;34(6):860-3.

43. Loureiro $\mathrm{J}$, et al. Autosomal dominant spastic paraplegias: a review of 89 families resulting from a portuguese survey. JAMA Neurol. 2013;70(4): 481-7.

44. Lu X, et al. Genetic analysis of SPG4 and SPG3A genes in a cohort of Chinese patients with hereditary spastic paraplegia. J Neurol Sci. 2014; 347(1-2):368-71.

45. Luo $Y$, et al. Mutation and clinical characteristics of autosomal-dominant hereditary spastic paraplegias in China. Neurodegener Dis. 2014;14(4): 176-83.

46. Shin JW, et al. Novel mutation in the ATL1 with autosomal dominant hereditary spastic paraplegia presented as dysautonomia. Auton Neurosci. 2014;185:141-3.

47. Yonekawa $\mathrm{T}$, et al. Extremely severe complicated spastic paraplegia $3 \mathrm{~A}$ with neonatal onset. Pediatr Neurol. 2014:51(5):726-9.

48. Khan TN, et al. Evidence for autosomal recessive inheritance in SPG3A caused by homozygosity for a novel ATL1 missense mutation. Eur J Hum Genet. 2014;22(10):1180-4.

49. Klein CJ, et al. Application of whole exome sequencing in undiagnosed inherited polyneuropathies. J Neurol Neurosurg Psychiatry. 2014;85(11): $1265-72$.

50. Ishiura $\mathrm{H}$, et al. Molecular epidemiology and clinical spectrum of hereditary spastic paraplegia in the Japanese population based on comprehensive mutational analyses. J Hum Genet. 2014;59(3):163-72.

51. Leonardi $L$, et al. De novo mutations in SPG3A: a challenge in differential diagnosis and genetic counselling. Neurol Sci. 2015;36(6):1063-4.

52. Park $\mathrm{H}$, et al. Mutational spectrum of the SPAST and ATL1 genes in Korean patients with hereditary spastic paraplegia. J Neurol Sci. 2015;357(1-2): $167-72$.

53. Zhao N, et al. Mutation analysis of four Chinese families with pure hereditary spastic paraplegia: pseudo-X-linked dominant inheritance and male lethality due to a novel ATL1 mutation. Genet Mol Res. 2015;14(4): 14690-7.

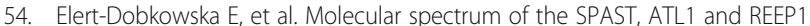
gene mutations associated with the most common hereditary spastic paraplegias in a group of Polish patients. J Neurol Sci. 2015;359(1-2):35-9.

55. Polymeris AA, et al. A series of Greek children with pure hereditary spastic paraplegia: clinical features and genetic findings. J Neurol. 2016;263(8): 1604-11.

56. Balicza P, et al. Genetic background of the hereditary spastic paraplegia phenotypes in Hungary-An analysis of 58 probands. J Neurol Sci. 2016; 364(1):116-21

57. Guelly C, et al. Targeted high-throughput sequencing identifies mutations in atlastin-1 as a cause of hereditary sensory neuropathy type I. Am J Hum Genet. 2011;88(1):99-105

58. Leonardis L, et al. The N355K atlastin 1 mutation is associated with hereditary sensory neuropathy and pyramidal tract features. Eur J Neurol. 2012;19(7):992-8.
59. Namekawa M, et al. A founder effect and mutational hotpots may contribute to the most frequent mutations in the SPG3A gene. Neurogenetics. 2016;7(2):131-2.

60. Sanderson CM, et al. Spastin and atlastin, two proteins mutated in autosomal-dominant hereditary spastic paraplegia, are binding partners. Hum Mol Genet. 2006;15(2):307-18

\section{Submit your next manuscript to BioMed Central and we will help you at every step:}

- We accept pre-submission inquiries

- Our selector tool helps you to find the most relevant journal

- We provide round the clock customer support

- Convenient online submission

- Thorough peer review

- Inclusion in PubMed and all major indexing services

- Maximum visibility for your research

Submit your manuscript at www.biomedcentral.com/submit 